\title{
Profile of pitolisant in the management of narcolepsy: design, development, and place in therapy
}

This article was published in the following Dove Press journal:
Drug Design, Development and Therapy
Abstract: Narcolepsy is a rare sleep disorder characterized by excessive daytime sleepiness and rapid eye movement sleep dysregulation, manifesting as cataplexy and sleep paralysis, as well as hypnagogic and hypnopompic hallucinations. Disease onset may occur at any age, although adolescents and young adults are mainly affected. Currently, the diagnosis delay ranges from 8 to 10 years and drug therapy may only attenuate symptoms. Pitolisant is a first-in-class new drug currently authorized by the European Medicines Agency to treat narcolepsy with or without cataplexy in adults and with an expanded evaluation for the treatment of neurologic diseases such as Parkinson's disease and epilepsy. This article reviews the pharmacokinetic and pharmacodynamic profile of pitolisant, highlighting its effectiveness and safety in patients with narcolepsy. We performed a systematic review of the literature using PubMed, Embase, and Google Scholar. We report on the efficacy and safety data of pitolisant in narcoleptic patients regarding cataplexy episodes and subjective and objective daytime sleepiness. The development program of pitolisant was characterized by eight Phase II/III studies. One proof-of-concept study followed by two pivotal studies, three randomized controlled trials, and two open studies were evaluated. Our review confirmed the effectiveness of pitolisant in treating major clinically relevant narcolepsy symptoms, including cataplexy, as compared to placebo. In addition, pitolisant revealed a safe profile when compared with placebo and active comparators. Headache, insomnia, and nausea were the prominent side effects. Further long-term randomized controlled trials comparing the efficacy of pitolisant with active comparators (ie, modafinil and sodium oxybate) may clarify its real place in therapy and its possible use as a first-line agent on the basis of its safety and tolerability.

Keywords: pitolisant, narcolepsy, cataplexy, histamine, excessive daytime sleepiness

\section{Introduction}

Narcolepsy is a rare sleep disorder characterized by excessive daytime sleepiness (EDS) and abnormal rapid eye movement (REM) sleep manifestations (ie, cataplectic attacks, sleep paralysis, hypnagogic, and hypnopompic hallucinations). This condition affects $0.026 \%-0.05 \%$ of the general population in North America and Europe. ${ }^{1}$ Disease onset may occur at any age, although adolescents and young adults are mainly affected..$^{2-4}$

The International Classification of Sleep Disorders third edition ${ }^{5}$ defines narcolepsy type 1 (NT1) and type 2 (NT2). NT1 is characterized by cataplexy and cerebrospinal fluid (CSF) orexin deficiency due to loss of orexinergic neurons in the lateral hypothalamus. ${ }^{6,7}$ The pathophysiology of the selective and substantial loss of orexinergic neurons is still unclear, although inflammatory/autoimmune processes seem to be highly involved..$^{8-10}$ The autoimmune hypothesis is supported by the large association of NT1 with human leukocyte antigen marker DQB $1 * 0602 .{ }^{11}$ In addition, recent findings have
Correspondence: Andrea Romigi Chief Sleep Medicine Center, IRCCS Neuromed, Via Atinense I8, Pozzilli (IS) 82077, Italy Tel +390865929679

Email andrea.romigi@gmail.com 
established an increased number of histaminergic neurons in NT1, suggesting involvement of the histamine system as a compensatory mechanism to orexin deficit. ${ }^{12}$ By contrast, no such increase has been detected in neurodegenerative disorders involving loss of orexinergic neurons, including Parkinson's disease, Alzheimer's disease, and Huntington disease. Both orexin and histamine have been implicated in sleep-wake regulation, and a reduction in histamine concentration in the CSF of narcoleptic subjects was previously described, ${ }^{12}$ although this finding has not been confirmed. ${ }^{13}$

EDS may represent the main presenting and often the most disabling symptom, with episodes of irresistible, typically short daily sleep that are associated with dreaming, followed by a feeling of being refreshed. In addition, NT1 patients complain of cataplexy (the sudden loss of muscle tone triggered by strong emotions), as well as frequent sleep paralysis, hypnagogic/hypnopompic hallucinations, disturbed night-time sleep with periodic leg movements, restless legs syndrome, ${ }^{14,15}$ and REM sleep behavior disorder. ${ }^{16}$ Further critical issues are represented by obesity, affecting about $30 \%$ of patients, and weight gain, which is frequently reported close to disease onset. ${ }^{17}$ Depression and anxiety are often reported as psychiatric comorbidities and they may further worsen the quality of life of narcoleptic patients. ${ }^{18}$ The clinical course of NT1 is chronic. EDS and cataplexy may improve over time; on the other hand, nocturnal sleep disturbance may worsen. NT2 is similar to NT1 except for the lack of cataplexy and orexin deficiency, although 10\%-20\% of non-cataplectic patients may show low CSF orexin levels and/or cataplexy may occur over time. ${ }^{19}$ Current therapy for narcolepsy may only attenuate clinical symptoms.

Recently, it has been demonstrated that hypothalamic orexinergic neurons (Hcrt) modulate histaminergic transmission by glutamate and orexin and inhibition of dynorphinmediated gamma-aminobutyric acid release. ${ }^{20,21}$ The Hcrt showed a neuromodulator role of sleep/wake cycle as reported in several studies. ${ }^{22-28}$

Pitolisant is a first-in-class drug acting on histamine H3 receptors (H3Rs) as an antagonist/inverse agonist. ${ }^{29}$ It enhances histaminergic neuron activation and other neurotransmitters in the brain (ie, dopamine, acetylcholine, noradrenaline).$^{30-32}$ Preclinical studies showed that pitolisant may enhance histamine and noradrenaline neuronal activity, increase wakefulness, and increase REM sleep latency in narcoleptic orexin knockout mice. ${ }^{33}$ The experimental data obtained in wild-type and orexin knockout mice and in cats demonstrate that pitolisant enhances wakefulness and reduces slow-wave sleep and REM sleep, thereby corroborating the possibility of using it to treat human narcolepsy. ${ }^{30,31}$ Since this mechanism of action is different from the current treatment, pitolisant may represent an alternative drug for narcoleptic patients. Pitolisant should be started at the lowest effective dose and titrated up to a maximal dosage of $36 \mathrm{mg}$ /day. The recommended regimens should range between 9 and $36 \mathrm{mg} /$ day, taken as a morning single dose. ${ }^{32,34}$ On Week 1 , the initial dose of $9 \mathrm{mg}$ /day should be proposed. On Week 2 , the dose may be increased to $18 \mathrm{mg} /$ day or decreased to $4.5 \mathrm{mg} /$ day. Finally, on Week 3 , the dose may be increased up to $36 \mathrm{mg} /$ day. At any time, the dose can be decreased (down to $4.5 \mathrm{mg} /$ day) or increased (up to $36 \mathrm{mg}$ /day) according to the physician assessment and the patient's response. Each tablet contains $5 \mathrm{mg}$ of pitolisant hydrochloride equivalent to $4.45 \mathrm{mg}$ of pitolisant. $^{34}$

\section{Literature search strategy}

Studies discussed in this paper were searched using the Embase, PubMed, and Google Scholar databases from inception to September 30, 2017. The following keywords were used: "pitolisant" and "narcolepsy", "cataplexy" and "treatment". The inclusion criteria were randomized controlled trials (RCTs) and open studies examining the use of pitolisant on subjects suffering from narcolepsy with or without cataplexy. Comparators, language, and publication status did not constitute the exclusion criteria. We also considered unpublished data from RCTs presented in the reports of regulatory agencies. Two authors (AR and VF) screened the search results independently.

\section{Pitolisant: design and development Chemical properties}

Pitolisant, formerly known as BF 2.649 and tripolisant, corresponds chemically to $1-\{3-[3-(4-c h l o r o p h e n y l) p r o p o x y]$ propyl \} piperidine hydrochloride (Figure 1). It is a non-chiral molecule with no stereoisomerism, available as a white/ almost white crystalline powder. Pitolisant is highly soluble in water, methylene chloride, and ethanol and insoluble in cyclohexane.

\section{Mechanism of action}

Pitolisant is a selective H3R antagonist/inverse agonist. H3Rs are mainly located in the brain and primarily expressed in the cerebral cortex, hypothalamus, hippocampus, and basal

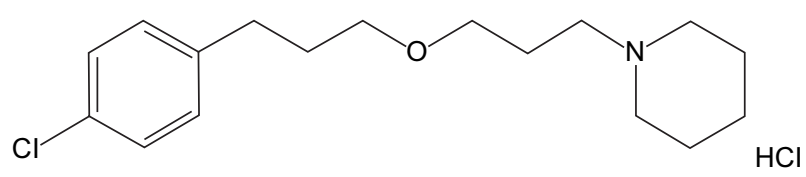

Figure I Chemical structure of pitolisant or I-\{3-[3-(4-chlorophenyl)propoxy] propyl\}piperidine hydrochloride. 
ganglia. ${ }^{35}$ The main action of pitolisant is mediated at a presynaptic level through activation of histaminergic neurons in the brain, which are involved in a large variety of functions including wakefulness, attention, and memory. In addition to the promotion of histamine release, pitolisant modulates the release of various neurotransmitters involved in wake promotion in the brain, such as dopamine, noradrenaline, and acetylcholine. ${ }^{36}$

\section{Pharmacokinetic properties}

In healthy volunteers, pitolisant exposure was established in $>200$ subjects after single oral doses up to $216 \mathrm{mg}$ and for a 28-day period. ${ }^{36}$ The gastrointestinal absorption of pitolisant is rapid, with peak plasma concentrations usually attained $3 \mathrm{~h}$ after oral administration. A double pitolisant dose increase from 27 to $54 \mathrm{mg}$ was associated with a 2.3-fold area under the concentration-time curve (AUC) increase from time zero to infinity. ${ }^{36}$

Pitolisant is highly bound to serum protein $(>90 \%)$ and is distributed fairly evenly between plasma and red blood cells. Pitolisant is eliminated mainly in urine (about $63 \%$ of the administered dose) and has a plasma half-life of $\sim 10-12 \mathrm{~h}$, reaching steady state within 5-6 days. Urine metabolites include BP2.951, which is a pharmacologically inactive and glycineconjugated metabolite. ${ }^{36}$ Available evidence on pitolisant metabolism indicates that many hydroxylated and conjugated derivatives are generated. The primary pharmacologically inactive metabolite is 5-aminovaleric acid recovered from serum and urine and mediated by cytochrome P450 (CYP) enzymes CYP3A4 and CYP2D6. Major conjugated metabolites include a glycuronidated ketone metabolite of monohydroxy desaturated pitolisant and a glycine conjugate of the acid metabolite of $O$-dealkylated desaturated pitolisant. In vitro studies suggested that pitolisant is a CYP3A4, CYP2B6, and CYP1A2 inducer and a CYP2D6 and OCT1 inhibitor. In vitro studies showed that pitolisant is not a substrate and has no inhibitory effect on breast cancer resistance protein and human P-glycoprotein. ${ }^{36}$ In elderly patients (aged 68-80 years), the pharmacokinetic parameters of pitolisant are not different from those observed in younger patients (aged 18-45 years). Mild hepatic dysfunction does not significantly affect pitolisant pharmacokinetics compared to that of healthy volunteers. However, an AUC increase by a factor of 2.4 and a double half-life were detected in patients with moderate hepatic impairment. In patients with renal disease (creatinine clearance $15-89 \mathrm{~mL} / \mathrm{min}$ ), $\mathrm{a} \mathrm{C}_{\text {max }}$ and AUC increase was noted with no impact on half-life. ${ }^{36}$

\section{Drug abuse liability}

Regarding the abuse potential, discrimination, and locomotor sensitization, conditioned-place preference, and self administration were evaluated in different animal models (rodents and primates), by different route of administration (oral, intraperitoneal, intravenous, subcutaneous), at different doses and with negative and positive control groups (modafinil, cocaine, and vehicle saline). The dependence potential of pitolisant was assessed in rats with morphine, cocaine, or amphetamine as positive references. These studies did not show evidence of any abuse or dependence potential on the part of pitolisant, except for the selfadministration study conducted in rhesus monkeys, as the highest pitolisant-tested dose served as a reinforcer for two out of four monkeys, though the obtained results were not conclusive. ${ }^{34,37}$ In addition, pitolisant showed affinity for sigma 1 (as agonist) and 2 receptors (as antagonist) with similar affinity for H3R. ${ }^{34}$ The reinforcing effects of sigma receptor agonists in rats with a history of cocaine self-administration has been previously reported, while some literature data reported the potential of sigma receptor antagonists as treatments for stimulant abuse. ${ }^{38}$ In addition, available studies indicated that sigma receptor agonists may induce an increase of dopamine concentration in rat nucleus accumbens shell. ${ }^{39}$ Thus, the available experimental data do not suggest any evident risk of abuse potential, but it could not be excluded. Furthermore, since pitolisant was found to enhance memory performance and the duration of acquisition in animal studies, diversion of pitolisant to improve intellectual performance has been considered as a potential risk in humans..$^{40,41}$

\section{Efficacy data}

Starting from a reliable experimental model of narcolepsy, Lin et $\mathrm{a}^{31}$ confirmed the hypothesis that enhancing histamine release in narcoleptic mice may circumvent orexin deficiency. ${ }^{30,42}$ These authors went on to conduct the first comparative single-blind, placebo-controlled, Phase II study to determine the potential effect of pitolisant on EDS in narcoleptic patients. ${ }^{31}$ The pitolisant development program in narcolepsy was characterized by eight Phase II/III studies (P07-03 and P09-15, respectively, Harmony I and Harmony Ibis were pivotal studies). In addition, two studies, P11-05 (Harmony CTP ${ }^{43}$ and P09-10 (Harmony III), were supportive for the evaluation by EMA. ${ }^{44}$ Key characteristics of the study designs and a summary of the main results are reported in Table 1.

\section{Phase II studies}

Lin et $\mathrm{a}^{31}$ performed the first proof-of-concept study of pitolisant in narcoleptic patients. This trial was a Phase II single-blind, multicenter, placebo-controlled study 


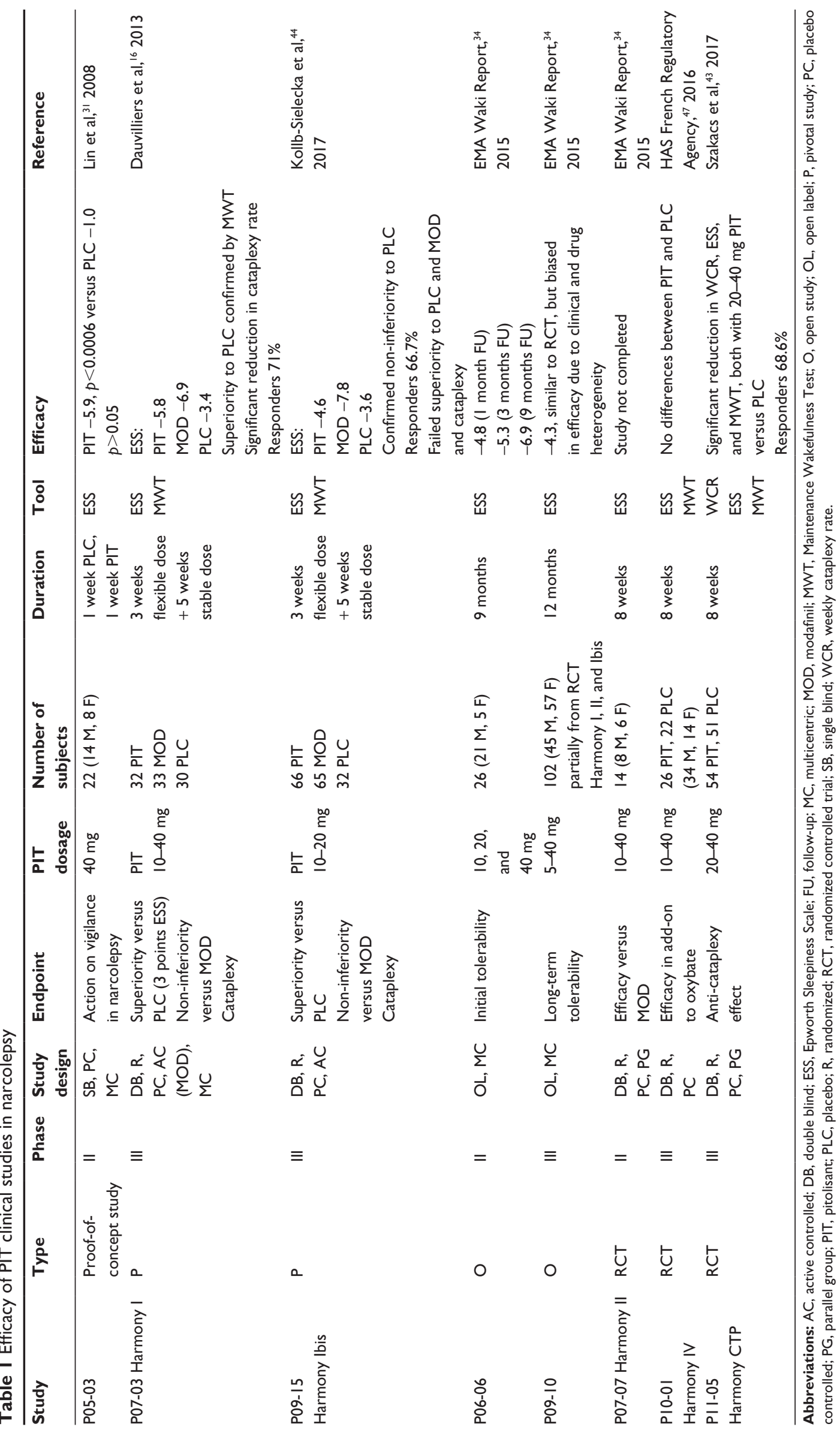


(Study P05-03). The authors selected 22 adult narcoleptic patients. All patients stopped psychostimulant treatments at least 3 days before inclusion; however, anti-cataplectic medications, at stable doses for at least 3 months, remained unchanged (except for tricyclic antidepressants which were withdrawn before the study due to their histamine $\mathrm{H} 1$ receptor antagonist activity and possible pharmacologic interactions with pitolisant). The main concomitant anti-cataplectic agent was venlafaxine ( $8 / 22$ patients, 36.3\%). Patients received placebo once a day for 1 week, followed by $40 \mathrm{mg}$ of pitolisant taken in the morning, $\sim \mathrm{h}$ after awakening, in the following week. The active treatment induced a significant reduction in the Epworth Sleepiness Scale (ESS) score from a baseline value of $17.55 \pm 3.89$ to $11.81 \pm 6.11$, with a $4.86 \pm 5.12$ point reduction ( $95 \%$ CI: 2.22 to $7.56 ; p=0.0006$ ) compared to placebo and a $5.85 \pm 5.51$ point reduction $(95 \%$ CI: 3.42 to $8.34 ; p<0.0001)$ versus baseline values, whereas with placebo, no significant decrease versus baseline was observed $(p>0.05) .^{44}$

In the P06-06 study, a multicenter, open-label, Phase II trial, pitolisant was evaluated in 26 narcoleptic patients in an escalating dose regimen of 10,20 , or $40 \mathrm{mg}$ /day given for up to 9 months. After 1, 3, and 9 months of treatment, the mean ESS scores were reduced by $4.8,5.3$, and 6.9 points, respectively.

The P07-07 study (Harmony II), a randomized, doubleblind, controlled, parallel-group trial to assess pitolisant efficacy in narcoleptic patients, compared a pitolisant group to pitolisant in add-on to modafinil. The study enrolled 14 patients and was stopped precociously. The subjects were subsequently enrolled in Harmony III, a supportive Phase III study (P09-10).

\section{Pivotal studies}

The efficacy of pitolisant in treating narcolepsy was demonstrated in two main, double-blind, 8-week, multicenter, parallel-group, placebo-controlled randomized trials. ${ }^{44}$ Each of these used a flexible dosing schedule. The starting dosage and the maximum dosage used were 9 and $36 \mathrm{mg} /$ day, respectively, in the Harmony I study and 4.5 and $18 \mathrm{mg} /$ day, respectively, in the Harmony Ibis study. The primary efficacy endpoint was defined as a minimal clinically relevant difference in the final ESS score between the pitolisant and placebo groups, with this value being set at 3 points. Eligible patients were aged 18 years and older, not taking psychostimulants for at least 14 days, and showing EDS (defined as an ESS score of 14 or more). The first pivotal $\mathrm{RCT}^{45}$ evaluated narcoleptic patients from 32 centers in Europe. Ninety-five out of 110 patients were randomized to pitolisant $(n=32)$, modafinil $(n=33)$, or placebo $(n=30)$ for 8 weeks: 3 weeks of flexible dosing $(10,20$, or $40 \mathrm{mg} /$ day of pitolisant and 100,200 , or $400 \mathrm{mg} /$ day of modafinil) followed by 5 weeks of stable dosage. Over the 8-week treatment period, the mean ESS score reductions were $-3.4 \pm 4.2$ in the placebo group, $-5.8 \pm 6.2$ in the pitolisant group, and $-6.9 \pm 6.2$ in the modafinil group. After adjustment for baseline, pitolisant proved its superiority to placebo (difference $-3.3,95 \%$ CI: -5.83 to $-0.83 ; p=0.024)$, but not its non-inferiority to modafinil (difference $0.12,95 \% \mathrm{CI}:-2.5$ to 2.7 ; $p=0.250$ ). In addition, the superiority of pitolisant compared to placebo was confirmed by the significant increase in the ability to stay awake ( $\mathrm{min}$ ) as measured by the Maintenance of Wakefulness Test (MWT; difference 1.47,95\% CI: 1.01 to $-2.14 ; p=0.044$ ), a validated tool to test the efficacy of sleepiness treatment in narcolepsy. ${ }^{45}$ Superiority of pitolisant over placebo was also confirmed by the higher responder rate on the ESS score (defined as ESS $\leq 10$ ) compared to placebo (odds ratio [OR] 9.25 [3.82 to 22.35]; $p<0.001$ ), while no statistical difference was detected between the pitolisant and modafinil responder rates (OR 1.06 [0.44 to 2.54]; $p=0.894$ ). The authors, therefore, concluded that pitolisant up to $40 \mathrm{mg}$ was efficacious on EDS compared with placebo, but not with modafinil.

The second pivotal RCT, Harmony Ibis ${ }^{44}$ evaluated narcoleptic patients randomly allocated to pitolisant $(n=67)$, modafinil $(n=65)$, or placebo $(n=33)$. The drug regimen involved increasing the pitolisant and modafinil doses: 3 weeks of flexible dosing according to clinical symptoms (10 and $20 \mathrm{mg} /$ day of pitolisant; 100, 200, $400 \mathrm{mg} /$ day of modafinil) followed by 5 weeks of stable dosage. Over the 8-week treatment period, the mean ESS score reductions were $-3.6 \pm 5.6$ in the placebo group, $-4.6 \pm 4.6$ in the pitolisant group, and $-7.8 \pm 5.9$ in the modafinil group. After adjustment for baseline, pitolisant failed to demonstrate superiority to placebo (difference $-1.94,95 \% \mathrm{CI}$ : -4.005 to $-0.07 ; p=0.06,<3$ points). However, non-inferiority to placebo was statistically achieved after reallocation of small centers (not preplanned analysis), even though the result was below the minimum clinically relevant difference of 3 points. A second planned analysis of non-inferiority of pitolisant compared to modafinil (up to $400 \mathrm{mg} /$ day) with a predefined non-inferiority margin threshold of 2 points was performed, resulting in rejection of non-inferiority of pitolisant compared to modafinil (difference $=-2.75,95 \%$ CI $[-4.48$ to -1.02$])$. Furthermore, when a superiority test comparing pitolisant to modafinil was performed (sensitivity analysis), modafinil showed significantly $(p<0.002)$ better results on the ESS final score ( $\Delta=-2.75$ points). The clinical efficacy of pitolisant over placebo was also confirmed by the higher ESS responder rate score (defined as ESS $\leq 10$ ) versus 
placebo (OR 9.24 [95\% CI: 3.82 to 22.35]; $p<0.001$ ), similar to the modafinil responder rate (OR 1.06 [0.44 to 2.54]; $p=0.894)$. Again, the responder rate on ESS scores according to responders' definition of Harmony Ibis (ESS $\leq 10$ or ESS baseline - ESS final $\geq 3$ points) demonstrated the superiority of pitolisant when compared to placebo (response rate $=0.60$ [95\% CI: 0.41 to 0.88 ]; $p<0.008$ ). Moreover, on a final visit, pitolisant significantly increased MWT versus placebo $(p<0.009)$, with an increase in the pitolisant group (delta $+1.14 \mathrm{~min}$ ) and a decrease in the placebo group (delta -1.39 min). Analysis of secondary endpoints, including MWT, Sustained Attention to Response Task Total, Clinical Global Impressions of Change scores, and responder rate, on EDS symptoms confirmed the positive efficacy data observed on the primary endpoint. The anti-cataplectic effects in both studies were not consistent. The Harmony I study showed a significant reduction in the cataplexy rate from baseline to final visit (baseline $>0.6$ cataplectic episodes versus final visit $<0.2$ episodes in the pitolisant group; $R R=0.38,95 \% \mathrm{CI}$ [0.16 to 0.93 ]; $p=0.034)$, whereas Harmony Ibis showed an increasing trend ( 0.84 episodes from baseline compared with 1.69 episodes at the final visit in the pitolisant group with no statistically significant difference versus placebo $p=0.077$ ). Key characteristics of the study designs and a summary of the main results are reported in Table 1. Anti-cataplectic effects of pitolisant are summarized in Figure 2.

\section{Phase III RCTs}

P11-05 (Harmony CTP) was a supportive double-blind, randomized, parallel-group study on pitolisant versus placebo in narcoleptic patients $(n=105$, age range $18-66$, mean age 34 years for the pitolisant group and 39 years for the placebo group). ${ }^{43}$ The patients included showed a high frequency of cataplexy attacks and an ESS score of 12 or more at baseline. The treatment phase lasted 7 weeks: 3 weeks of flexible dosing $(5,10$, or $20 \mathrm{mg}$ once daily) followed by 4 weeks of stable dosing $(5,10,20$, or $40 \mathrm{mg}$ once daily). The primary endpoint was the effect of pitolisant on cataplexy expressed as a change in the average number of cataplexy attacks per week between the 2-week baseline period and the 4-week treatment period. This study confirmed the beneficial effect of pitolisant on cataplexy. The primary analysis showed a significant improvement in the pitolisant group at the end of the stable dose treatment period. The weekly cataplexy rate between the 2 weeks of baseline and the 4-week stable dosing period decreased from 7.31 to 6.79 in the placebo group and from 9.15 to 3.28 for pitolisant, with the ratio rate for pitolisant/placebo being $0.512(95 \% \mathrm{CI}: 0.435$ to $0.603 ; p<0.0001)$. At the end of treatment, the percentage of patients showing a high cataplexy rate (weekly cataplexy rate $>15$ ) was significantly higher in the placebo group $(23.5 \%[12 / 51])$ than in the pitolisant group (5.6\% [3/54]; $p<0.0001$ ). Both $20 \mathrm{mg}$ (9 patients) and $40 \mathrm{mg}$ (35 patients) induced a significant decrease in the cataplexy rate $(20 \mathrm{mg}$, from 8.42 to $3.38 ; 40 \mathrm{mg}$, from 8.46 to 3.57 ) as compared to placebo (from 7.09 to 6.28). In addition, this study confirmed a significant improvement in EDS symptoms induced by pitolisant versus placebo as measured by mean ESS scores (mean changes placebo $-1.9 \pm 4.3$ and pitolisant $-5.4 \pm 4.3$; $p<0.001)$. The mean change in ESS was clinically relevant

\section{Anticataplectic effect of pitolisant}

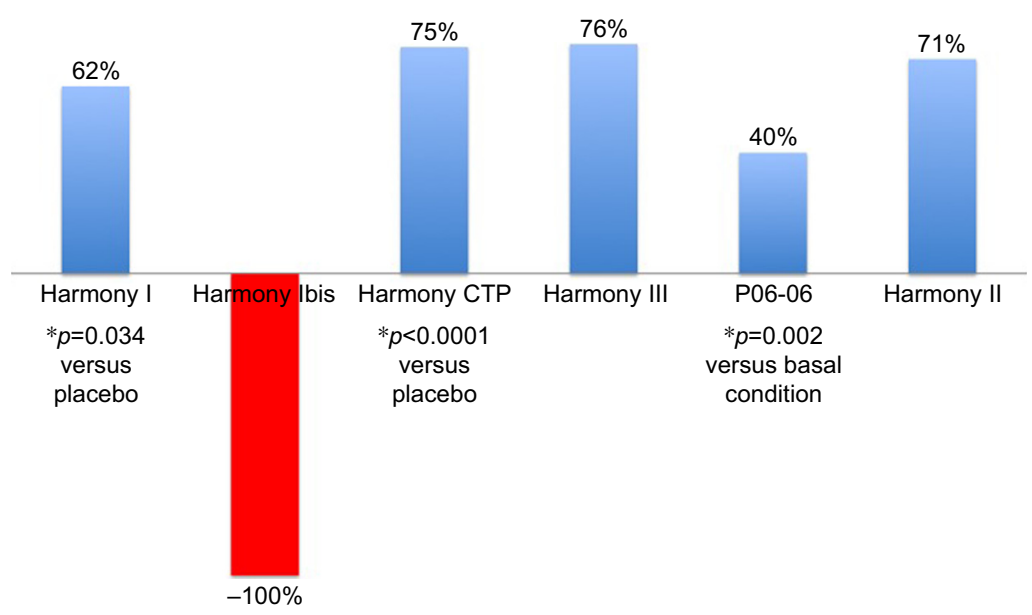

Figure 2 Anti-cataplectic effect of pitolisant expressed as cataplexy reduction (\%) in the main clinical trials.

Notes: Harmony lbis only did not show cataplexy reduction. In this study, the maximum pitolisant dose was limited to $18 \mathrm{mg} /$ day (reached by $75 \%$ of patients). In addition, an important imbalance between the study sites of Harmony lbis led to comparison of results with or without cluster grouping of sites. Cataplexy rates in Harmony lbis were also not consistent with those seen in Harmony I. ${ }^{32}$ Harmony III study reported $76 \%$ reduction in total cataplexy and $64 \%$ reduction in partial cataplexy. ${ }^{46} * p<0.05$ statistically significant. Abbreviation: PL, placebo. 
(above 3 units) in the pitolisant group. The results obtained from MWT confirmed the improvement in alertness as expressed by the geometric mean of the ratios (MWT final/ MWT baseline) for pitolisant compared to placebo that was 1.8 (95\% CI: 1.19 to $2.71 ; p=0.005)$. This observation was confirmed by the aggregated $z$ score (combining ESS and MWT), showing a final $z$ score of $0.97(\mathrm{SD}=1.35)$ in the pitolisant group compared with $0.22(\mathrm{SD}=1.29)$ in the placebo group. This study confirmed that the efficacy of pitolisant on EDS was comparable to that observed in Harmony I, a trial on less-severe narcoleptic patients with the same maximal dosage (40 mg/day). The data from Harmony CTP supported previous data from the pivotal studies.

The last RCT was the P10-01 (Harmony IV) study, a randomized, double-blind, 8-week, placebo-controlled, add-on to sodium oxybate in 48 narcoleptic patients. The study is completed, but data have not been published, although the French National Authority for Health ${ }^{47}$ included data from the study in the report of the Commission de la Transparence regarding pitolisant (21 June 2016). The inclusion and exclusion criteria for this study were similar to those of Harmony I and Ibis. The initial dose was $5 \mathrm{mg}$, with a gradual increase up to $40 \mathrm{mg} /$ day during the first 5 weeks. The dose had then to be maintained for 1 month. No difference in daytime sleepiness assessed by the ESS (primary endpoint) was found between the two groups: at the end of the study, the mean score decreased by 2.6 points in the pitolisant group and 2.1 points in the placebo group $(p=0.595)$. No differences were found between pitolisant and placebo in addition to sodium oxybate treatment on the secondary endpoints, especially on the MWT, reduction of cataplexy attacks, and quality of life. ${ }^{47}$

\section{Long-term studies}

P09-10 (Harmony III) was a Phase III, open-label, prospective, longitudinal, uncontrolled, multicenter trial designed to evaluate the long-term safety of pitolisant in the treatment of EDS in narcolepsy. ${ }^{44}$ The study enrolled 102 adult narcoleptic patients with or without cataplexy who had completed a double-blind controlled study with pitolisant (Harmony I, the prematurely stopped Harmony II, Harmony Ibis, or other Phase II studies in narcolepsy), patients who would be unable to participate in a double-blind study but could benefit from pitolisant, or patients receiving pitolisant under the French compassionate use program. The duration of the study was 12 months, and the maximal dose received was $36 \mathrm{mg} /$ day in $88 \%$ of patients. The open-label design, the lack of reference therapy, and the concomitant presence of drug-naïve and already treated patients did not allow for unbiased conclusions on efficacy, even though open-label, naturalistic, and observational study may be considered a good alternative and accepted as the "best possible evidence" of efficacy for rare diseases. ${ }^{48}$ However, a comparison between the effect of pitolisant observed in the naturalistic study and the effects observed in pivotal trials confirmed the maintenance of clinical efficacy over a longer period, as demonstrated by the ESS change from baseline to final visit ( -4.3 points), similar to those obtained in Harmony I ( -5.8 points) and Harmony Ibis ( -4.6 points). Similarly, the responder rate $(\mathrm{ESS} \leq 10$ or final $\mathrm{ESS}-$ basal ESS $\geq 3$ ) was of the same magnitude as in Harmony Ibis (68.2\%), using the same definition of responders. In addition, cataplexy reduction rate $(76 \%)$ was similar to that obtained in short-term study (Harmony I 62\%; Harmony CTP 75\%; P06-06 40\%; Harmony II 71\%), as reported in Figure 2.

\section{Safety}

Although pitolisant treatment was generally well tolerated, its safety profile cannot be compared with active drugs with similar pharmacodynamics because it is the first antagonist/ inverse agonist H3R utilized in clinics. ${ }^{44}$

Safety was assessed mainly from data obtained in narcolepsy studies, but also from RCTs in different clinical contexts (Parkinson's disease and sleep apnea related to EDS, epilepsy, dementia, attention-deficit/hyperactivity disorder, schizophrenia). ${ }^{44}$ At this moment, the clinical program for pitolisant involves 1,385 subjects ( 291 healthy controls and 1,094 patients, of whom 342 were suffering from narcolepsy, while the remaining 742 patients were being treated for different indications). The median exposure time was 64 days for narcoleptic patients, and the cumulative duration of exposure was higher than 1 year in 219/1,094 (20\%), ranging between 6 months and 1 year in 150/1,094 (14\%) and was $<6$ months in 725/1,094 (66\%). In narcolepsy, the percentage of patients treated with pitolisant $(52.3 \%)$ who reported at least one adverse event (AE) was slightly higher than placebo $(41.1 \%)$ and similar to a different active drug (modafinil, 55.1\%). The AE profile of pitolisant in narcolepsy was similar to other indications. ${ }^{44}$

As a consequence of the mechanism of action, the most frequent AEs were neuropsychiatric effects. Psychiatric disorders were reported more frequently with pitolisant $(21.9 \%)$ than with placebo (8.9\%) and modafinil (13.3\%). Gastrointestinal AEs were also more frequent with pitolisant $(16.1 \%)$ than with placebo (8.2\%). Conversely, central nervous system disorders were slightly less frequently reported 
in pitolisant groups $(22.8 \%$ versus $23.5 \%$ modafinil versus 20.9\% placebo).

From pooled analysis, the most frequent AEs in narcoleptic patients proved to be mild headache $(16.1 \%$ versus modafinil $12.2 \%$ and placebo $12.7 \%$ ), insomnia ( $7.6 \%$ versus placebo $1.9 \%$ ), anxiety (3.5\% versus modafinil 3.1\%), depression $(2.3 \%$ versus modafinil $1 \%)$, irritability $(3.2 \%$ versus modafinil $3.1 \%$ and placebo $0.6 \%)$, dizziness $(2.3 \%$ versus modafinil $5.1 \%$ and placebo $2.5 \%)$, hallucinations $(1.8 \%$ versus modafinil 1\%) and vertigo (1.2\%). Other AEs were nausea (5.6\% versus modafinil $2 \%$ and placebo $3.2 \%$ ), weight increase $(2.9 \%$ versus placebo $1.3 \%)$, vomiting $(2.3 \%)$, and diarrhea ( $2 \%$ versus modafinil $6.1 \%$ and placebo $1.9 \%) .{ }^{31,33,49}$ The most frequent side effects of pitolisant versus modafinil and placebo in narcoleptic patients, pooled from all studies, are reported in Table 2 and Figure 3.

The available data do not allow any clear conclusions concerning a weight modification profile of pitolisant. Even though, as previously said, weight gain was reported in $2.9 \%$ of narcoleptic patients, weight decrease was also reported, albeit less frequently. ${ }^{44}$ Obesity and weight gain were considered not so much a simple comorbidity, but an integral part of narcoleptic orexinergic dysregulation. ${ }^{50}$ Hence, neurologists should carefully monitor significant body weight changes. Similarly, as depression and anxiety were frequently reported in narcolepsy (ranging from 15\% to $37 \%),{ }^{51}$ it may be difficult to find a causal relationship between treatment and these psychiatric AEs. However, the recent report by the EMA on pitolisant and narcolepsy advised caution in subjects with a history of psychiatric disorders and suicidal risk ideation.

Less-common AEs were hot flushes, leg pain, hallucinations, apathy, lack of appetite, abdominal discomfort,

Table 2 Tolerability of pitolisant: AEs from all studies pooled

\begin{tabular}{llll}
\hline AE & $\begin{array}{l}\text { Pitolisant } \\
\%(\mathbf{n}), \mathbf{n}=\mathbf{3 4 2}\end{array}$ & $\begin{array}{l}\text { Modafinil } \\
\%(\mathbf{n}), \mathbf{n}=\mathbf{9 8}\end{array}$ & $\begin{array}{l}\text { Placebo } \\
\%(\mathbf{n}), \mathbf{n}=\mathbf{I} \mathbf{5 8}\end{array}$ \\
\hline Headache & $\mathrm{I} 6 . \mathrm{I}(55)$ & $\mathrm{I} 2.2(\mathrm{I} 2)$ & $\mathrm{I} 2.7(20)$ \\
Insomnia & $7.6(26)$ & $\mathrm{NR}$ & $\mathrm{I} .9(3)$ \\
Nausea & $5.6(\mathrm{I})$ & $2(2)$ & $3.2(5)$ \\
Weight increase & $2.9(\mathrm{I})$ & $\mathrm{NR}$ & $\mathrm{I} .3(2)$ \\
Anxiety & $3.5(\mathrm{I})$ & $3 . \mathrm{I})(3)$ & $\mathrm{NR}$ \\
Depression & $2.3(8)$ & $\mathrm{I}(\mathrm{I})$ & $\mathrm{NR}$ \\
Vomiting & $2.3(8)$ & $\mathrm{NR}$ & $\mathrm{NR}$ \\
Irritability & $3.2(\mathrm{II})$ & $3 . \mathrm{I}(3)$ & $0.6(\mathrm{I})$ \\
Diarrhea & $2(7)$ & $6 . \mathrm{I}(6)$ & $\mathrm{I} .9(3)$ \\
Dizziness & $2.3(8)$ & $5 . \mathrm{I}(5)$ & $2.5(4)$ \\
Back pain & $2.3(8)$ & $\mathrm{NR}$ & $0.6(\mathrm{I})$ \\
Nasopharyngitis & $2(7)$ & $6.1(6)$ & $0.6(\mathrm{I})$ \\
\hline
\end{tabular}

Abbreviations: $A E$, adverse event; NR, not reported.

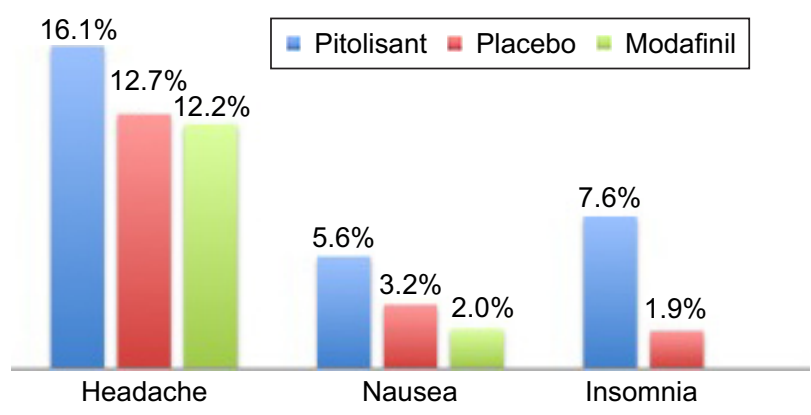

Figure 3 Most common adverse events (\% of treated patients) reported for pitolisant, modafanil, and placebo pooled from all studies (insomnia was not reported for modafanil in pooled studies).

diarrhea, sweating, and malaise. No withdrawal syndrome was detected following pitolisant treatment.

\section{Pitolisant and narcolepsy: place in therapy}

Narcolepsy is a rare, life-long, neurologic disease for which there is currently no cure and the pharmacologic treatment is still puzzling. Although behavioral therapy may be considered the most effective approach, patients' quality of life is significantly affected by both daytime somnolence and cataplexy. ${ }^{52}$ Present treatment options may relieve the main narcoleptic symptoms (ie, EDS and cataplexy) and, to a lesser extent, lessen sleep disruption, hypnagogic/hypnopompic hallucinations, and sleep paralysis. Sodium oxybate is considered as a first-line agent for cataplexy and EDS, and may help sleep disruption, hypnagogic hallucinations, and sleep paralysis. Modafinil and its $R$-enantiomer armodafinil are considered first-line agents for the treatment of diurnal somnolence, although neither agent affects cataplexy. ${ }^{53}$

Antidepressant agents including norepinephrine serotonin reuptake inhibitors (ie, venlafaxine, duloxetine), ${ }^{54,55}$ selective serotonin reuptake inhibitors (eg, fluoxetine), ${ }^{56}$ norepinephrine reuptake inhibitor (eg, reboxetine), ${ }^{57}$ and tricyclic agents (protriptyline, imipramine, or clomipramine) $)^{58}$ and monoamine oxidase B inhibitors (selegiline) ${ }^{59}$ represent the second-line agents for treating cataplexy. ${ }^{52}$ However, periodic leg movements in sleep, restless legs syndrome, and REM sleep behavior disorders are frequent sleep comorbidities in narcoleptic patients that may be exacerbated by sodium oxybate and/or antidepressant intake, and may require a modification in treatment, behavioral procedures, and iron addition when serum ferritin levels are reduced. ${ }^{52,60}$

Sodium oxybate showed clinical efficacy in NT1 and its use seems to be effective and safe, ${ }^{61}$ although the potential risks of abuse, diversion, uncertain tolerability, and a narrow safety margin are still debated. Furthermore, sodium oxybate should not be prescribed in combination with other sedatives, 
respiratory depressants, or muscle relaxants, restrictions that are well known to sleep experts. ${ }^{62-64}$ Nevertheless, treatment options for NT1 and NT2 are inadequate and induce partial relief of symptoms. ${ }^{52}$

Pitolisant is a first-in-class drug acting as an antagonist/ inverse agonist of the H3Rs, and its mechanism of action is novel and distinctive compared to the currently available therapies. Very recently, the EMA recognized that the benefits of pitolisant used to treat narcoleptic patients outweighed the risks and approved this drug for the treatment of NT1 and NT2. ${ }^{34,44}$ As previously reported, pitolisant demonstrated efficacy on excessive daytime somnolence and cataplexy in short-term studies when compared with placebo. ${ }^{44}$

In addition, the anti-cataplectic effects were recently confirmed in a well-designed randomized, double-blind, placebo-controlled trial conducted in 105 patients suffering from narcolepsy with cataplexy; a reduction in the mean frequency of cataplexy was shown. ${ }^{43}$

Thus, pitolisant has been approved in the European Union as treatment of narcolepsy with and without cataplexy and recognized as an orphan drug by the EMA and US Food and Drug Administration. Pitolisant may be considered as a promising first-line option for daytime somnolence and cataplexy, even though the short-term duration of the studies, the lack of data demonstrating the non-inferiority of pitolisant versus active drugs and/or in case of failure or intolerance to alternative drugs (ie, modafinil, sodium oxybate, and methylphenidate) should be taken into account as well-recognized issues of rare diseases. ${ }^{43,48,65}$ Although the non-inferiority compared to modafinil, as measured by means of ESS score changes, failed to be demonstrated in pivotal studies, the historical comparison from six RCTs with modafinil, to which the Harmony I and Ibis studies were added, indicates that the effect of pitolisant on EDS in narcolepsy, when used up to $40 \mathrm{mg} /$ day, is of the same degree as modafinil. ${ }^{34}$ Long-term efficacy data could not be obtained from Harmony III due to efficacy conclusions being biased by open design, no reference treatment, coexistence of drug-naïve and treated subjects, and the use of simultaneous psychostimulant drugs. ${ }^{34,44}$

However, the available data have allowed the approval of pitolisant for clinical use in a rare and orphan condition such as narcolepsy with and without cataplexy. As most narcoleptic patients treated with pitolisant were diagnosed based on the International Classification of Sleep Disorders, second edition criteria, ${ }^{66}$ the use of more recent and specific criteria should improve the quality of confirmatory studies regarding the effect of pitolisant on cataplexy and EDS. ${ }^{19}$
Further long-term RCTs comparing the effectiveness of pitolisant with active drugs (modafinil, sodium oxybate) are strongly called for to clarify its real place in therapy and its probable utility as a first-line agent, considering its safe and tolerable profile.

\section{Disclosure}

Andrea Romigi has received speaker honoraria from EISAI Europe and UCB Pharma. Giuseppe Vitrani has received speaker honorarium from Fidia Pharma. The other authors have no conflicts of interest to disclose.

\section{References}

1. Ohayon MM, Priest RG, Zulley J, Smirne S, Paiva T. Prevalence of narcolepsy symptomatology and diagnosis in the European general population. Neurology. 2002;58(12):1826-1833.

2. Wijnans L, Lecomte C, de Vries C, et al. The incidence of narcolepsy in Europe: before, during, and after the influenza A(H1N1)pdm09 pandemic and vaccination campaigns. Vaccine. 2013;31(8):1246-1254.

3. Oberle D, Drechsel-Bauerle U, Schmidtmann I, Mayer G, KellerStanislawski B. Incidence of Narcolepsy in Germany. Sleep. 2015; 38(10):1619-1628.

4. Dauvilliers Y, Montplaisir J, Molinari N, et al. Age at onset of narcolepsy in two large populations of patients in France and Quebec. Neurology. 2001;57(11):2029-2033.

5. American Academy of Sleep Medicine. International Classification of Sleep Disorders. 3rd ed. Darien, IL: American Academy of Sleep Medicine; 2014.

6. Peyron C, Faraco J, Rogers W, et al. A mutation in a case of early onset narcolepsy and a generalized absence of hypocretin peptides in human narcoleptic brains. Nat Med. 2000;6(9):991-997.

7. Thannickal TC, Moore RY, Nienhuis R, et al. Reduced number of hypocretin neurons in human narcolepsy. Neuron. 2000;27(3): 469-474.

8. Partinen M, Kornum BR, Plazzi G, Jennum P, Julkunen I, Vaarala O. Does autoreactivity have a role in narcolepsy? Lancet Neurol. 2014; 13(11):1072-1073.

9. Liguori C, Placidi F, Albanese M, et al. CSF beta-amyloid levels are altered in narcolepsy: a link with the inflammatory hypothesis? J Sleep Res. 2014;23(4):420-424.

10. Liguori C, Placidi F, Izzi F, et al. Beta-amyloid and phosphorylated tau metabolism changes in narcolepsy over time. Sleep Breath. 2016; 20(1):277-283.

11. Scammell TE. Narcolepsy. N Engl J Med. 2015;373(27):2654-2662.

12. Nishino S, Sakurai E, Nevsimalova S, et al. Decreased CSF histamine in narcolepsy with and without low CSF hypocretin-1 in comparison to healthy controls. Sleep. 2009;32(2):175-180.

13. Dauvilliers Y, Delallee N, Jaussent I, et al. Normal cerebrospinal fluid histamine and tele-methylhistamine levels in hypersomnia conditions. Sleep. 2012;35(10):1359-1366.

14. Plazzi G, Ferri R, Antelmi E, et al. Restless legs syndrome is frequent in narcolepsy with cataplexy patients. Sleep. 2010;33(5):689-694.

15. Dauvilliers Y, Pennestri MH, Petit D, Dang-Vu T, Lavigne G, Montplaisir J. Periodic leg movements during sleep and wakefulness in narcolepsy. J Sleep Res. 2007;16(3):333-339.

16. Dauvilliers Y, Jennum P, Plazzi G. Rapid eye movement sleep behavior disorder and rapid eye movement sleep without atonia in narcolepsy. Sleep Med. 2013;14(8):775-781.

17. Kotagal S, Krahn LE, Slocumb N. A putative link between childhood narcolepsy and obesity. Sleep Med. 2004;5(2):147-150.

18. Pizza F, Magnani M, Indrio C, Plazzi G. The hypocretin system and psychiatric disorders. Curr Psychiatry Rep. 2014;16(2):433. 
19. Ito E, Inoue Y. [The International Classification of Sleep Disorders, third edition. American Academy of Sleep Medicine. Includes bibliographies and index]. Nihon Rinsho. 2015;73(6):916-923. Japanese.

20. Schone C, Burdakov D. Glutamate and GABA as rapid effectors of hypothalamic "peptidergic" neurons. Front Behav Neurosci. 2012;6:81.

21. Alexandre C, Andermann ML, Scammell TE. Control of arousal by the orexin neurons. Curr Opin Neurobiol. 2013;23(5):752-759.

22. Morairty SR, Dittrich L, Pasumarthi RK, et al. A role for cortical nNOS/ NK1 neurons in coupling homeostatic sleep drive to EEG slow wave activity. Proc Natl Acad Sci U S A. 2013;110(50):20272-20277.

23. de Lecea L, Huerta R. Hypocretin (orexin) regulation of sleep-to-wake transitions. Front Pharmacol. 2014;5:16.

24. Vetrivelan R, Qiu MH, Chang C, Lu J. Role of Basal Ganglia in sleep-wake regulation: neural circuitry and clinical significance. Front Neuroanat. 2010;4:145.

25. Simon C, Kezunovic N, Ye M, et al. Gamma band unit activity and population responses in the pedunculopontine nucleus. J Neurophysiol. 2010;104(1):463-474.

26. Monti JM. The role of dorsal raphe nucleus serotonergic and non-serotonergic neurons, and of their receptors, in regulating waking and rapid eye movement (REM) sleep. Sleep Med Rev. 2010;14(5):319-327.

27. Lin JS, Sergeeva OA, Haas HL. Histamine H3 receptors and sleep-wake regulation. J Pharmacol Exp Ther. 2011;336(1):17-23.

28. Carter ME, Yizhar O, Chikahisa S, et al. Tuning arousal with optogenetic modulation of locus coeruleus neurons. Nat Neurosci. 2010;13(12): 1526-1533.

29. Schwartz JC. The histamine H3 receptor: from discovery to clinical trials with pitolisant. Br J Pharmacol. 2011;163(4):713-721.

30. Ligneau X, Landais L, Perrin D, et al. Brain histamine and schizophrenia: potential therapeutic applications of H3-receptor inverse agonists studied with BF2.649. Biochem Pharmacol. 2007;73(8):1215-1224.

31. Lin JS, Dauvilliers Y, Arnulf I, et al. An inverse agonist of the histamine $\mathrm{H}(3)$ receptor improves wakefulness in narcolepsy: studies in orexin-/- mice and patients. Neurobiol Dis. 2008;30(1):74-83.

32. Syed YY. Pitolisant: first global approval. Drugs. 2016;76(13): 1313-1318.

33. Inocente $\mathrm{C}$, Arnulf I, Bastuji $\mathrm{H}$, et al. Pitolisant, an inverse agonist of the histamine $\mathrm{H} 3$ receptor: an alternative stimulant for narcolepsycataplexy in teenagers with refractory sleepiness. Clin Neuropharmacol. 2012;35(2):55-60.

34. (CHMP) EMACfMPfHU. Assessment report Wakix International nonproprietary name: pitolisant. Procedure No. EMEA/H/C/002616/0000 19th November 2015. Available from: http://www.ema.europa.eu/ docs/en_GB/document_library/EPAR_-_Public_assessment_report/ human/002616/WC500204749.pdf. 2015. Accessed November 20, 2017.

35. Martinez-Mir MI, Pollard H, Moreau J, et al. Three histamine receptors $(\mathrm{H} 1, \mathrm{H} 2$ and $\mathrm{H} 3)$ visualized in the brain of human and non-human primates. Brain Res. 1990;526(2):322-327.

36. Wakix. Summary of product characteristics. Available from: http:// www.ema.europa.eu/docs/en_GB/document_library/EPAR_Product_Information/human/002616/WC500204746.pdf. Accessed October $15,2017$.

37. Uguen M, Perrin D, Belliard S, et al. Preclinical evaluation of the abuse potential of Pitolisant, a histamine $\mathrm{H}_{3}$ receptor inverse agonist/antagonist compared with Modafinil. Br J Pharmacol. 2013;169(3):632-644.

38. Narayanan S, Mesangeau C, Poupaert JH, McCurdy CR. Sigma receptors and cocaine abuse. Curr Top Med Chem. 2011;11(9):1128-1150.

39. Katz JL, Hong WC, Hiranita T, Su TP. A role for sigma receptors in stimulant self-administration and addiction. Behav Pharmacol. 2016; 27(2-3 Spec Issue):100-115.

40. Brabant $\mathrm{C}$, Charlier Y, Tirelli E. The histamine $\mathrm{H}_{3}$-receptor inverse agonist pitolisant improves fear memory in mice. Behav Brain Res. 2013; 243:199-204

41. Sadek B, Saad A, Sadeq A, Jalal F, Stark H. Histamine $\mathrm{H}_{3}$ receptor as a potential target for cognitive symptoms in neuropsychiatric diseases. Behav Brain Res. 2016;312:415-430.
42. Ligneau X, Perrin D, Landais L, et al. BF2.649 [1-\{3-[3-(4-Chlorophenyl)propoxy]propyl piperidine, hydrochloride], a nonimidazole inverse agonist/antagonist at the human histamine $\mathrm{H}_{3}$ receptor: preclinical pharmacology. J Pharmacol Exp Ther. 2007;320(1):365-375.

43. Szakacs Z, Dauvilliers Y, Mikhaylov V, et al; HARMONY-CTP study group. Safety and efficacy of pitolisant on cataplexy in patients with narcolepsy: a randomised, double-blind, placebo-controlled trial. Lancet Neurol. 2017;16(3):200-207.

44. Kollb-Sielecka M, Demolis P, Emmerich J, Markey G, Salmonson T, Haas M. The European medicines agency review of pitolisant for treatment of narcolepsy: summary of the scientific assessment by the committee for medicinal products for human use. Sleep Med. 2017;33: 125-129.

45. Dauvilliers Y, Bassetti C, Lammers GJ, et al; HARMONY I study group. Pitolisant versus placebo or modafinil in patients with narcolepsy: a double-blind, randomised trial. Lancet Neurol. 2013;12(11): 1068-1075.

46. Schwartz JC, Lecomte JM, Caussé C. Anticataplectic efficacy of wakix $^{\circledR}$ (pitolisant), the first potent and highly selective histamine h3-receptor antagonist/inverse agonist in clinics. Sleep Med. 2017; 40(S1):e297.

47. HAS HADS. Commission de la Transparence. Available from: https:// www.has-sante.fr/portail/upload/docs/evamed/CT-14970_WAKIX_ PIC_INS_Avis3_CT14970.pdf 2016. Accessed November 20, 2017.

48. Clarke JT, Coyle D, Evans G, Martin J, Winquist E. Toward a functional definition of a "rare disease" for regulatory authorities and funding agencies. Value Health. 2014;17(8):757-761.

49. Dauvilliers Y, Barateau L. Narcolepsy and other central hypersomnias. Continuum (Minneap Minn). 2017;23(4, Sleep Neurology):989-1004.

50. Ponziani V, Gennari M, Pizza F, Balsamo A, Bernardi F, Plazzi G. Growing up with type 1 narcolepsy: its anthropometric and endocrine features. J Clin Sleep Med. 2016;12(12):1649-1657.

51. Vandeputte M, de Weerd A. Sleep disorders and depressive feelings: a global survey with the Beck depression scale. Sleep Med. 2003;4(4): 343-345.

52. Abad VC, Guilleminault C. New developments in the management of narcolepsy. Nat Sci Sleep. 2017;9:39-57.

53. Morgenthaler TI, Kapur VK, Brown T, et al; Standards of Practice Committee of the American Academy of Sleep Medicine. Practice parameters for the treatment of narcolepsy and other hypersomnias of central origin. Sleep. 2007;30(12):1705-1711.

54. Moller LR, Ostergaard JR. Treatment with venlafaxine in six cases of children with narcolepsy and with cataplexy and hypnagogic hallucinations. J Child Adolesc Psychopharmacol. 2009;19(2):197-201.

55. Izzi F, Placidi F, Marciani MG, et al. Effective treatment of narcolepsycataplexy with duloxetine: a report of three cases. Sleep Med. 2009; 10(1):153-154

56. Frey J, Darbonne C. Fluoxetine suppresses human cataplexy: a pilot study. Neurology. 1994;44(4):707-709

57. Larrosa O, de la Llave Y, Bario S, Granizo JJ, Garcia-Borreguero D. Stimulant and anticataplectic effects of reboxetine in patients with narcolepsy: a pilot study. Sleep. 2001;24(3):282-285.

58. Touchon J. [Use of antidepressants in sleep disorders: practical considerations]. Encephale. 1995;21 Spec No 7:41-47. French.

59. Mayer G, Ewert Meier K, Hephata K. Selegeline hydrochloride treatment in narcolepsy. A double-blind, placebo-controlled study. Clin Neuropharmacol. 1995;18(4):306-319.

60. St Louis EK. Key sleep neurologic disorders: Narcolepsy, restless legs syndrome/Willis-Ekbom disease, and REM sleep behavior disorder. Neurol Clin Pract. 2014;4(1):16-25.

61. Black J, Pardi D, Hornfeldt CS, Inhaber N. The nightly use of sodium oxybate is associated with a reduction in nocturnal sleep disruption: a double-blind, placebo-controlled study in patients with narcolepsy. J Clin Sleep Med. 2010;6(6):596-602.

62. Lammers GJ, Bassetti C, Billiard M, et al. Sodium oxybate is an effective and safe treatment for narcolepsy. Sleep Med. 2010;11(1):105-106. 
63. Zvosec DL, Smith SW, Hall BJ. Three deaths associated with use of Xyrem. Sleep Med. 2009;10(4):490-493.

64. Boscolo-Berto R, Viel G, Montagnese S, Raduazzo DI, Ferrara SD, Dauvilliers Y. Narcolepsy and effectiveness of gamma-hydroxybutyrate (GHB): a systematic review and meta-analysis of randomized controlled trials. Sleep Med Rev. 2012;16(5):431-443.
65. Kallweit U, Bassetti CL. Pharmacological management of narcolepsy with and without cataplexy. Expert Opin Pharmacother. 2017; 18(8):809-817.

66. Baumann CR. Wide implications of a trial on pitolisant for cataplexy. Lancet Neurol. 2017;16(3):173-174.

\section{Publish your work in this journal}

Drug Design, Development and Therapy is an international, peerreviewed open-access journal that spans the spectrum of drug design and development through to clinical applications. Clinical outcomes, patient safety, and programs for the development and effective, safe, and sustained use of medicines are the features of the journal, which has also been accepted for indexing on PubMed Central. The manuscript management system is completely online and includes a very quick and fair peer-review system, which is all easy to use. Visit http://www.dovepress.com/testimonials.php to read real quotes from published authors.

Submit your manuscript here: http://www.dovepress.com/drug-design-development-and-therapy-journal 